\title{
Tratamiento de la anemia con eritropoietina y hierro en Enfermedad Renal Crónica
}

\author{
MÓNICA CUEVAS P. ${ }^{1}$, PÍA ROSATI M. ${ }^{2}$, FRANCISCO CANO S. ${ }^{3}$
}

1. Médico Pediatra, Instructor Escuela de Medicina Universidad de Chile. Becada del Programa de Especialista en Nefrología Infantil, Escuela de Postgrado, Universidad de Chile.

2. Nefrólogo Pediatra. Hospital San Juan de Dios.

3. Nefrólogo Pediatra, Profesor Asociado, Unidad de Nefrología Hospital Luis Calvo Mackenna. Facultad de Medicina Universidad de Chile.

\begin{abstract}
Erythropoietin and iron in anemia management in chronic renal failure

Anemia is one of the most common manifestations of Chronic Renal Failure (CRF), specially during the dialysis period. Growth failure and a high cardiovascular morbimortality are 2 of the most important consequences. Objective: To present a review of the current concepts in diagnosis and management of anemia in pediatric CRF patients. Erythropoietin (EPO) deficit is the main cause of anemia, requiring exogenous replacement through intravenous or subcutaneous route, in hemodialyzed or peritoneodialyzed patients respectively. A longer half-life allows to use EPO one or twice weekly when given by intraperitoneal route, in order to reach a target hemoglobin between 11-12 $\mathrm{gr} / \mathrm{dl}$, a level that avoids the cardiovascular risk associated to higher levels as described in adult CRF population. In pediatrics, $100-300 \mathrm{U} / \mathrm{kg} /$ weekly can be used to reach the desired hemoglobin levels, always monitoring about the potential complications of EPO, specially arterial hypertension. If anemia seems to be resistent to EPO therapy, iron deficit should be considered and properly treated, as described in this article. Ferritin and saturation of hemoglobin need to be routinely monitored to diagnose iron status in these patients, values less than $100 \mathrm{ng} / \mathrm{ml}$ and $20 \%$ respectively require exogenous suplementation. Iron doses in pediatrics still need to be defined. Anemia needs to be evaluated and treated in all CRF children, properly management must always be instaured in order to prevent its undesired effects.
\end{abstract}

(Key words: Anemia, erythropoietin, iron, chronic renal failure, dialysis).

Rev Chil Pediatr 2008; 79 (2): 131-145

\section{RESUMEN}

La anemia es una de las complicaciones más frecuentes en los pacientes pediátricos con Enfermedad Renal Crónica (ERCR), especialmente en etapa de diálisis. Sus consecuencias van desde un aumento de la

Trabajo recibido el 27 de febrero de 2008, devuelto para corregir el 17 de marzo de 2008, segunda versión el 31 de marzo de 2008, aceptado para publicación el 5 de abril de 2008.

Correspondencia a:

Mónica Cuevas P.

E-mail:mcuevas@med.uchile.cl. 
morbilidad cardiovascular, hasta retraso del crecimiento en algunas experiencias analizadas. Se presenta una revisión de las recomendaciones actuales del diagnóstico, evaluación y tratamiento de la anemia en ERCR. Su principal causa es el déficit de la hormona Eritropoietina, la cual debe ser administrada en forma exógena para lograr una respuesta óptima. En los pacientes en hemodiálisis (HD) se prefiere la vía intravenosa, una dosis en cada sesión de HD, dado su mejor tolerancia, aunque esta vía requiere dosis mayores, su vida media es más corta, y representa costos más elevados. En niños en diálisis peritoneal (DP) la vía de elección es subcutánea, de mayor duración que la vía i.v., y puede ser administrada 1-2 veces por semana. En pediatría, dosis de mantención entre 100 - $300 \mathrm{U} / \mathrm{kg} / \mathrm{semana}$ han demostrado ser eficaces para lograr una hemoglobina entre 11 y 12 gr/dl, nivel considerado seguro desde el punto de vista cardiovascular, requiriendo sin embargo, una estricta supervisión médica dado las potenciales complicaciones, entre las que destaca la hipertensión arterial de difícil manejo y que requiere disminución o suspensión de la terapia. Entre las causas más frecuentes e importantes de una respuesta terapéutica insuficiente está el déficit en los depósitos de fierro del organismo. Estos depósitos se consideran adecuados al presentar el paciente una ferritina $>100 \mathrm{ng} / \mathrm{ml}$ y una saturación de hemoglobina $>20 \%$. Bajo estos valores se hace necesario el aporte exógeno para lograr una ferritina ideal entre 200 y 500 ng/ml, aporte cuya dosificación en el caso de niños en diálisis está aún por definirse. Se recomienda que el control de exámenes para evaluar anemia y la respuesta al tratamiento se realice, al menos, de forma trimestral.

(Palabras clave: anemia, eritropoietina, enfermedad renal crónica, hierro, diálisis).

Rev Chil Pediatr 2008; 79 (2): 131-145

\section{Introducción}

La anemia es una de las alteraciones más frecuentes en los pacientes con Enfermedad Renal Crónica (ERCR), siendo su etiología multifactorial. Entre las principales causas podemos destacar una eritropoiesis inadecuada ya sea por un déficit en la producción de eritropoyetina, depósitos de hierro insuficientes, estados inflamatorios crónicos (mediadores inflamatorios interfieren con la maduración de los precursores de glóbulos rojos en la médula ósea), hiperparatiroidismo severo (determina la aparición de mielofibrosis que puede obliterar la médula ósea) y/o procesos infecciosos intercurrentes. También contribuyen al desarrollo de la anemia una menor vida media de los glóbulos rojos, el déficit de vitaminas hidrosolubles, la administración de medicamentos citotóxicos o inmunosupresores y el uso de IECA $^{1,2}$. Lamentablemente, a diferencia de lo que ocurre en población adulta, no existen evidencias directas que permitan establecer un punto de corte preciso en la velocidad de filtración glomerular, a partir del cual aumente significativamente el riesgo de anemia ${ }^{2}$, por lo cual las recomendaciones actuales sugieren que todos los pacientes portadores de ERCR, independiente de la etapa, sean evaluados para identificar si existe anemia y así, poder iniciar un tratamiento apropiado lo más precozmente posible $^{3}$.

En los últimos años el tratamiento de la anemia en esta patología ha mejorado sustancialmente debido al uso regular de agentes estimuladores de eritropoiesis y suplementos de hierro. Entre los beneficios de un tratamiento adecuado de la anemia destacan una mejoría en la calidad de vida de los pacientes ${ }^{4}$, disminución del número de transfusiones de glóbulos rojos $^{1}$, efectos cardiovasculares (regresión de hipertrofia ventricular izquierda) ${ }^{5}$ y mejoría de la capacidad funcional e intelectual ${ }^{6,7}$. En pediatría se ha comunicado igualmente que la anemia podría contribuir al retraso del crecimiento que presentan estos pacientes ${ }^{4,5}$. A pesar de lo anterior, la anemia sigue siendo un problema de alta prevalencia en esta población. Así, por ejemplo, de acuerdo al último reporte de ESAM (European Survey on Anemia Management) sólo en un 66\% de la población estudiada (8100 pacientes adultos en tratamiento dialítico provenientes de 11 países Europeos e Israel) se alcanzaron niveles de hemoglobina 
mayores de $11 \mathrm{gr} / \mathrm{dl}^{8}$. En nuestro país, la Rama de Nefrología de la Sociedad Chilena de Pediatría reportó que un $82,6 \%$ de pacientes en hemodiálisis (HD), un 45,1\% de pacientes en DP y un 34\% de pacientes en tratamiento médico de ercr presentaban niveles de hemoglobina menores de 11,5 gr/dl ${ }^{[9]}$. En el mismo sentido, el ESRD CPM Project (End Stage Renal Disease Clinical Performance Measures Project) reportó que en menores de 18 años, un 34\% de los pacientes en HD y que un $31 \%$ de los pacientes en DP presentaban valores bajo $11 \mathrm{gr} / \mathrm{dl}^{10}$.

En relación al tratamiento, según el último reporte del Estudio cooperativo de trasplante renal pediátrico de Norteamérica (NAPRTCS 2006), al mes de iniciada la HD, un 59\% de los pacientes recibía hierro oral, 24\% hierro intravenoso y $94 \%$ eritropoietina. De la misma manera, considerando el grupo en DP un $74 \%$ recibía terapia oral, un 3\% parenteral y un 88\% eritropoietina ${ }^{11}$.

El tratamiento de la anemia en Enfermedad renal crónica (ERCR) adquiere una especial relevancia, dado que tanto en adultos ${ }^{12,13}$ como en población infantil se ha observado que la presencia de anemia es un predictor importante de morbi-mortalidad. Utilizando la base datos de NAPRTCS para identificar pacientes que ingresaron por primera vez a terapia dialítica entre los años 1992 y 2001, Warady y cols, determinaron que la presencia de anemia al mes de iniciada la diálisis aumentaba en un $52 \%$ el riesgo de muerte con un riesgo relativo de 1,52 (IC 95\% 1,03-2,226, p = 0,037); adicionalmente observaron que incrementaba el riesgo de hospitalizaciones más prolongadas. Es destacable que las principales causas de muerte en el grupo estudiado fueron patologías cardio-pulmonares e infecciosas ${ }^{14}$. En este sentido, dos estudios descriptivos han evaluado la relación existente entre anemia e hipertrofia ventricular izquierda (HVI) en niños con ERCR $^{15,16}$. En uno de ellos, se evaluó en forma retrospectiva a 64 pacientes en terapia dialítica detectándose que un $75 \%$ presentaba HVI, la que era significativamente más frecuente en pacientes en hemodiálisis. Los pacientes con HVI severa (41\%) presentaban niveles significativamente menores de hemoglobina $(\mathrm{p}=0,027)$ y un tiempo más prolongado de enfermedad renal previo a la diálisis comparado con pacientes sin HVI.

El objetivo de esta revisión ha sido realizar una actualización respecto al estudio y tratamiento de la anemia en pacientes portadores de Enfermedad renal crónica.

\section{Definición y evaluación de la anemia en ERCR}

El diagnóstico de anemia se establece con niveles de hemoglobina $(\mathrm{Hb})$ por debajo del percentil 5 para edad y sexo. En la actualidad se prefiere utilizar el valor de Hemoglobina por sobre el Hematocrito (Hcto) para el diagnóstico y control de la anemia en ERCR, ya que los resultados del hematocrito pueden verse afectados con mayor facilidad por técnicas de conservación de la muestra; así por ejemplo, su valor aumenta con mayor temperatura o duración del almacenaje, debido a que se produce edema de los glóbulos rojos. Por otra parte, la medición de $\mathrm{Hb}$ se realiza en forma directa, mientras que la determinación del Hcto es resultado de un cálculo matemático; finalmente, la $\mathrm{Hb}$ presenta menor disminución dilucional, es decir disminuye menos en situaciones de sobrecarga de volumen del paciente.

La evaluación etiológica de la anemia debe ser realizada de manera sistematizada utilizando elementos clínicos y de laboratorio. Una detallada historia clínica y examen físico que incluya la historia familiar debe ser realizada en todos los pacientes. En relación al estudio de laboratorio, las últimas guías de la National Kidney Foundation (NKF), denominadas KDOQI (Kidney Disease Outcome Quality Initiative $)^{17}$ indican que la evaluación inicial de estos pacientes debe considerar hemograma completo con recuento de reticulocitos, ferritina sérica para objetivar los depósitos de hierro y saturación de transferrina para evaluar el hierro disponible para eritropoiesis. Se recomienda que los depósitos de hierro sean evaluados mensualmente después de iniciar el tratamiento con agentes estimuladores de eritropoiesis hasta que el paciente se encuentre estable, posteriormente realizar controles al menos cada 3 meses. 
Las características de los glóbulos rojos determinadas por el volumen corpuscular medio (VCM) y la concentración media de hemoglobina corpuscular (CHCM) nos pueden orientar acerca de la etiología de la anemia. La anemia de la ERCR generalmente es normocítica, normocrómica e hiporegenerativa. Microcitosis e hipocromía es la presentación clásica de la anemia por déficit de hierro y la macrocitosis se asocia a déficit de Vitamina B12 y/o ácido fólico. Finalmente, el recuento de reticulocitos nos permite evaluar la respuesta medular frente a la anemia. Así, por ejemplo, depósitos de hierro adecuados asociados a un bajo recuento de reticulocitos pueden reflejar una producción inadecuada de eritropoietina. En la figura 1 se presenta un esquema del enfrentamiento del paciente con anemia en ercr.

Como se mencionó previamente, la principal causa de anemia en ercr es una síntesis deficiente de eritropoyetina por las células peritubulares intersticiales. La segunda causa en importancia es el déficit de hierro. Se han descrito tres tipos de déficit de hierro:

Absoluto, funcional y bloqueo inflamatorio; cuyas características se describen en la tabla $1^{2}$.

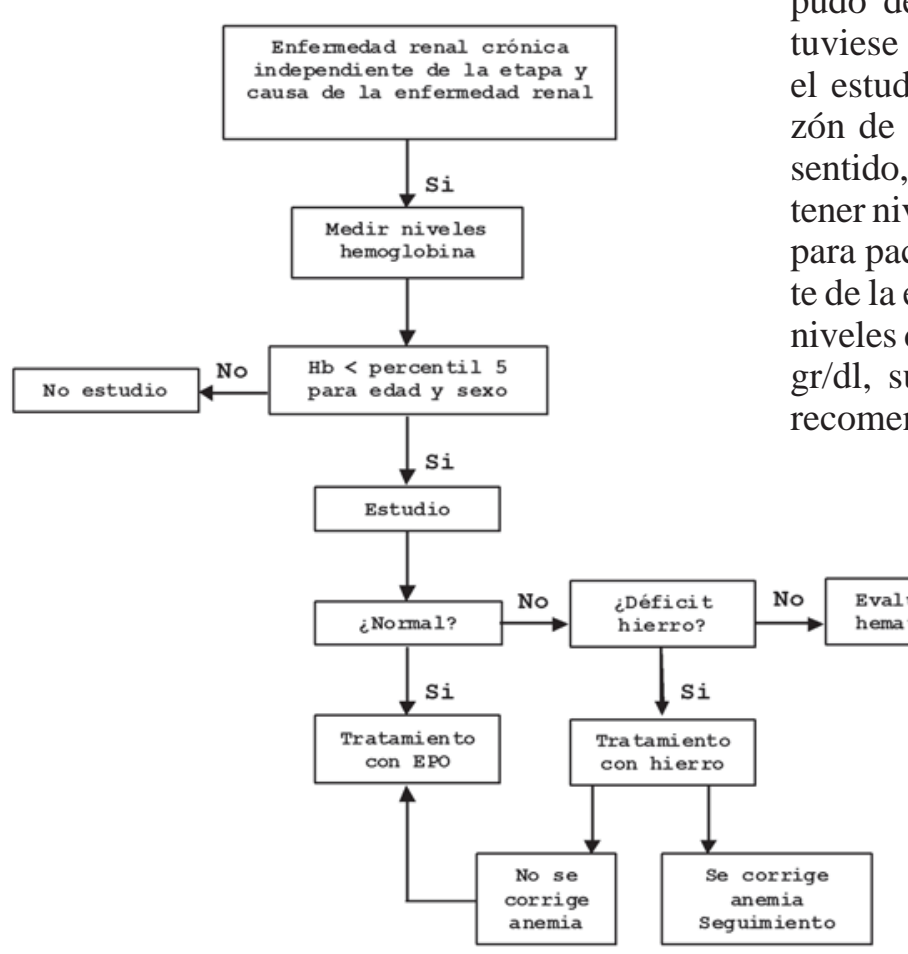

Figura 1. Enfrentamiento inicial de la anemia en el paciente con ERCR.

\section{Objetivos del tratamiento}

\section{Nivel de hemoglobina}

Se recomienda aumentar la hemoglobina a una velocidad de $1 \mathrm{gr} / \mathrm{dl}$ por mes, para mantener un rango que no represente complicaciones para el paciente. Este rango se ha definido por algunos autores entre 11-12 gr de $\mathrm{Hb} / \mathrm{dl}$, o un hematocrito entre 33-36\%, ya que valores por sobre dicho rango presentarían complicaciones sin mayores beneficios ${ }^{18,19}$. Singh A. y cols, en una fase del estudio CHOIR (Correction of Hemogloblin and Outcomes in Renal Insufficiency ${ }^{28}$ estudiaron en forma controlada, aleatoria y randomizada a 1432 pacientes adultos portadores de ercr para comparar el beneficio y seguridad de mantener una hemoglobina de 13,5 vs un valor de 11,3 mg/dl. La variable dependiente fue una combinación de muerte, accidente vascular encefálico y falla miocárdica, con un tiempo medio de seguimiento de 16 meses. En forma significativa, se observaron 125 eventos en el grupo de $\mathrm{Hb}$ 13,5 vs 97 eventos en el grupo de hemoglobina baja. En otro aspecto, usando un score destinado a analizar la calidad de vida de estos pacientes ( $\mathrm{Li}$ near Analogue Self-Assessment, LASA) no se pudo demostrar que alguno de los 2 grupos tuviese diferencias significativas al respecto, $\mathrm{y}$ el estudio fue cerrado anticipadamente en razón de la seguridad de los pacientes. En este sentido, las guías KDOQI $2007^{20}$ sugieren mantener niveles de hemoglobina entre 11 y $12 \mathrm{gr} / \mathrm{dl}$ para pacientes portadores de ercr independiente de la etapa (recomendación) y opinan que los niveles de hemoglobina no deben superar los 13 $\mathrm{gr} / \mathrm{dl}$, sugerencia que plantean en calidad de recomendación la cual debiera ser modificada 
Tabla 1. Tipos de déficit de hierro

\begin{tabular}{|c|c|c|c|}
\hline Forma & Indicador & Causa & $\begin{array}{l}\text { Respuesta al tratamiento } \\
\text { con hierro }\end{array}$ \\
\hline Déficit absoluto de hierro & $\begin{array}{l}\text { TSAT }<20 \% \text { y } \\
\text { Ferritina sérica }<100 \mathrm{ng} / \mathrm{mL}\end{array}$ & $\begin{array}{l}\uparrow \text { de pérdida } \\
\downarrow \text { absorción }\end{array}$ & $\begin{array}{l}\uparrow \text { TSAT y ferritina sérica } \\
\uparrow \text { hemoglobina }\end{array}$ \\
\hline Déficit funcional de hierro & $\begin{array}{l}\text { TSAT }<20 \% \text { y ferritina } \\
\text { sérica } 100-800 \mathrm{ng} / \mathrm{mL}\end{array}$ & $\begin{array}{l}\text { Estimulación intensa de } \\
\text { producción GR por } \\
\text { tratamiento con EPO } \\
\text { superando la oferta de hierro } \\
\text { Depósitos de hierro normal } \\
\text { o aumentado. }\end{array}$ & $\begin{array}{l}\uparrow \text { hemoglobina } \\
\downarrow \text { requerimientos EPO }\end{array}$ \\
\hline Bloqueo inflamatorio & $\begin{array}{l}\text { Dramático } \uparrow \text { de ferritina } \\
\text { sérica junto con } \downarrow \text { TSAT }\end{array}$ & $\begin{array}{l}\text { Inflamación aguda o crónica } \\
\text { Bloqueo de entrega de } \\
\text { depósitos de hierro del SRE }\end{array}$ & Sin respuesta \\
\hline
\end{tabular}

SER: sistema retículo-endotelial.

en las próximas guías. Adicionalmente, destacan que considerando la variabilidad en los valores de hemoglobina para edad y sexo y las variables de crecimiento y desarrollo propios de la edad pediátrica, no existen estudios suficientes para fijar valores de hemoglobina target en calidad de evidencia, por lo cual proponen mantener las recomendaciones de la población adulta.

\section{Parámetros de cinética de hierro}

Las recomendaciones actuales sugieren mantener niveles de ferritina sérica $>100 \mathrm{ng} / \mathrm{ml}$ y un porcentaje de saturación de transferrina $>20 \%$ en pacientes pediátricos en hemodiálisis, diálisis peritoneal o en tratamiento médico. En estudios realizados en adultos el límite inferior de ferritina sérica se incrementó de 100 a 200 $\mathrm{ng} / \mathrm{ml}$ basados en evidencia que sugiere que niveles de $100 \mathrm{ng} / \mathrm{ml}$ subestiman el déficit de hierro. Considerando que al respecto no existen estudios controlados y randomizados en población pediátrica, las guías actuales recomiendan mantener el límite inferior en $100 \mathrm{ng} / \mathrm{ml}$ hasta que exista mayor evidencia acerca de los riesgos y beneficios de mantener niveles de ferritina mayores. La sensibilidad y especificidad de la ferritina sérica para el diagnóstico de ferropenia en ercr es de un 48 y $75 \%$ para una ferritina sérica de $100 \mathrm{ng} / \mathrm{ml}, 77 \%$ y 37\% para una ferritina sérica de 200 ng/ml y de $90 \%$ y 18\% para una ferritina sérica de $300 \mathrm{ng} / \mathrm{ml}$, respectivamente.
De acuerdo a estas cifras se ha recomendado tomar como valor de corte para el diagnóstico de ferropenia un nivel de ferritina sérica de $100 \mathrm{ng} / \mathrm{ml}$ para situaciones de bajo riesgo clíni$\mathrm{co}$, y hasta $300 \mathrm{ng} / \mathrm{ml}$ en aquellos casos en que se considere importante aumentar la sensibilidad diagnóstica del test, como por ejemplo en pacientes con resistencia a la terapia con $\mathrm{EPO}^{20,21}$.

De acuerdo a lo mencionado, es opinión de los autores que en pacientes en HD sería recomendable mantener niveles de ferritina sobre $200 \mathrm{ng} / \mathrm{ml}$, ya que este grupo de pacientes presenta mayores pérdidas de sangre y tiene con mayor frecuencia anemia. Con respecto al límite superior de ferritina, las recomendaciones vigentes no apoyan la administración de hierro intravenoso si el nivel de ferritina es superior a $500 \mathrm{ng} / \mathrm{ml}$.

\section{Eritropoietina}

La hormona Eritropoietina (EPO) es una glicoproteína de 165 aminoácidos y 14 residuos de ácido siálico que regula la proliferación y diferenciación de los precursores eritroides en la médula ósea, consta de 3 cadenas de carbohidratos con enlace $\mathrm{N}$-asparaginasa, una con enlace O-serina, y un peso molecular (PM) de $31 \mathrm{kda}^{22}$. Es producida fundamentalmente por el riñón, órgano del que depende más del 90\% de su producción excepto durante la vida fetal y neonatal, períodos en que es básicamente producida por el hígado. 
Originalmente identificada en la década del 50 ' por Erslev y cols ${ }^{23}$, este hallazgo fue seguido por la identificación y clonación del gen correspondiente en el brazo largo del cromosoma 7, lo cual permitió la síntesis de la hormona recombinante en la década del 70', siendo usada por primera vez en la práctica clínica en el año $1986^{24}$.

Su producción depende del estímulo de la hipoxia en las células tubulares renales, fundamentalmente a partir de la caída del hematocrito. El aumento de la afinidad al oxígeno por parte de la hemoglobina o la presencia del monóxido de carbono con disminución de la entrega de oxígeno a nivel tisular, llevan igualmente a un aumento en la síntesis y liberación de la hormo$\mathrm{na}^{25}$.

Su disponibilidad se encuentra severamente comprometida en la enfermedad renal crónica, dado que el parénquima renal dañado produce una cantidad de hormona muy por debajo de las requeridas para una eritropoiesis normal, por lo que se hace necesaria su introducción en forma progresiva como hormona sintética recombinante.

La EPO existe en 2 formas para uso clínico, EPO alfa y EPO beta, con diferencias farmacocinéticas importantes y para uso intravenoso (i,v.) o subcutáneo (s.c.).

Las características farmacocinéticas de EPO alfa y EPO beta se detallan en la tabla 2. En general, la vía subcutánea permite obtener una vida media más larga, y eventualmente una disminución de la dosis semanal en un 30-50\% respecto a la vía i.v. Sin embargo, independiente de la vía de administración, los pacientes pediátricos requieren dosis por kg más elevadas en comparación con la población adulta, lo que parece ser secundario a un mayor clearance del medicamento y un mayor volumen de distribución de la EPO durante la niñez ${ }^{26,27}$.
Existen igualmente las EPO de acción prolongada (long-lasting EPO), entre las que se conoce desde hace algunos años la Darbepoietina alfa, una glicoproteína elaborada por ingeniería genética, cuya vida media es de $25 \pm 2$ y $48 \pm 5$ hrs al ser usada vía i.v. o s.c. Respectivamente, y que ha sido empleada con buenos resultados en pacientes urémicos adultos en modelos de aplicación cada 1-2 semanas ${ }^{28,29}$, en un régimen de conversión en el cual se reemplazan 200 U de EPO clásica por $1 \mathrm{mcg}$ de Darbepoietina alfa, aunque existen comunicaciones recientes que muestran que para dosis mayores de EPO la conversión a Darbepoietina puede ser menor, recomendando equivalencias de 1: 250 a 1: 350 en pacientes con dosis de EPO superiores a $5000 \mathrm{U}$ por sema$\mathrm{na}^{30}$. Recientemente se ha introducido en clínica la eritropoietina C.E.R.A. (Continuous Erythropoietin Receptor Activator) péptido con la misma estructura química de la EPO, al cual se le ha agregado una cadena de polietilenglicol (PEG) que aumenta su PM a 61 kda (figura 2), otorgándole una vida media de 133 y 137 horas

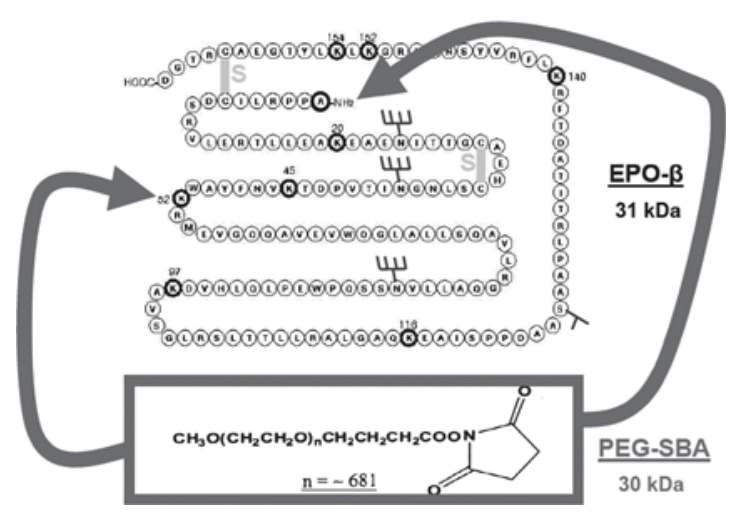

Figura 2. Esquema de la estructura molecular de EPOC.E.R.A.

Tabla 2. Características farmacocinéticas de EPO alfa y beta según vía de administración

\begin{tabular}{llccc} 
& EPO alfa i.v & EPO alfa s.c. & EPO beta i.v. & EPO beta s.c \\
\hline Concentración plasmática máxima & 15 minutos & $4-24 \mathrm{hrs}$ & $17 \mathrm{~min}$ & $12-28 \mathrm{hrs}$ \\
Biodisponibilidad & $100 \%$ & $25-30 \%$ & $100 \%$ & $23-42 \%$ \\
Vida media & $16 \mathrm{hrs}$ & $24 \mathrm{hrs}$ & $8,8 \mathrm{hrs}$ & $13-28 \mathrm{hrs}$ \\
Excreción & urinaria & urinaria & urinaria & urinaria \\
\hline
\end{tabular}




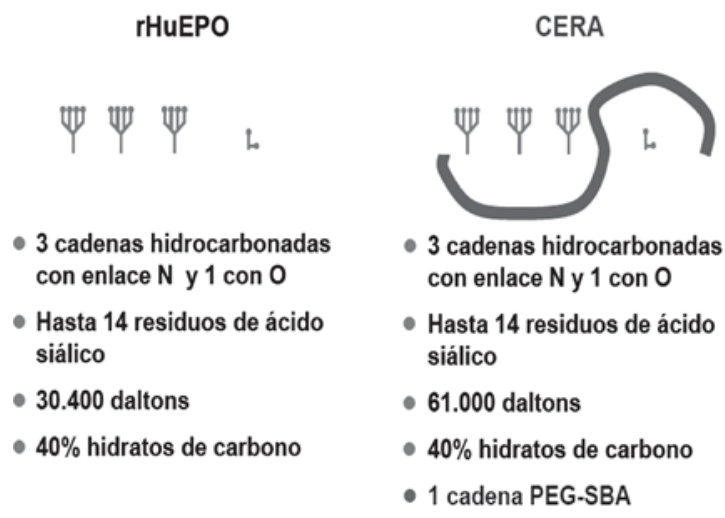

Figura 3. RHuEPO vs EPO-C.E.R.A.

tras una aplicación i.v. o s.c. Respectivamente, con estudios de fase III que muestran su eficacia en la corrección de la anemia en pacientes adultos urémicos al ser aplicado cada 2-4 semanas $^{31,32}$. En la figura 3 se realiza una comparación entre EPO recombinante humana y EPO C.E.R.A.

\section{Dosis y vía de administración}

La recomendación $\mathrm{KDOQI}^{3,33}$, lo mismo que la sugerida por las European Best Practice Guidelines $^{34}$, es administrar la EPO en pacientes en diálisis por vía subcutánea. Sin embargo, el grupo de trabajo de NK-KDOQI favorece la administración i.v. De EPO en pacientes que se encuentran en HD. En relación a la frecuencia de su uso, se recomienda iniciar en forma bisemanal en pacientes en diálisis peritoneal o trisemanal en hemodiálisis ${ }^{33 .}$

Las dosis requeridas en pediatría son mayores que en adultos en enfermedad renal crónica, debido a un mayor volumen de distribución corporal y una depuración más rápida, y se ha observado que a menor edad del paciente los requerimientos de EPO son mayores ${ }^{1,26,35}$, aunque la dosis inicial en niños puede ser difícil de determinar debido a diferencias individuales en la sobrevida eritrocitaria y a la capacidad remanente de producir EPO endógena. La dosis en pacientes en etapas pre-diálisis es menor que en diálisis (etapa 5), pudiendo iniciarse el tratamiento con 25-50 U/kg/semana en etapa 4, y 50-100 U/kg/semana en etapa $5^{26}$. Las recomendaciones de dosificación de EPO según
Tabla 3. Dosis de Eritropoietina (EPO) en la fase de mantención en pacientes pediátricos en diálisis

\begin{tabular}{lc}
\hline Peso & Dosis de EPO en U/kg/semana \\
\hline$>30 \mathrm{~kg}$ & 120 \\
$20-30 \mathrm{~kg}$ & 225 \\
$<20 \mathrm{~kg}$ & 300 \\
\hline
\end{tabular}

(adaptado de ref 26)

peso corporal se detallan en la tabla 3. Luego la dosis debe ser ajustada de acuerdo a la hemoglobina deseada, mediante un aumento de 25-50 U/kg/ semana para su uso s.c. o i.v. Respectivamente. La mayoría de los pacientes pediátricos responde a dosis entre 100-200 U/kg/ semana, dependiendo de una serie de factores como son el estado de los depósitos de fierro, la pérdida sostenida de eritrocitos en HD o por vía gastrointestinal, presencia de infecciones o de un estado inflamatorio crónico, y la edad del paciente $^{1,3,26}$.

En el informe NAPRTCS 2006 para 5256 pacientes pediátricos incluidos desde el año 1992 en adelante $^{11}$, el $95.8 \%$ de los pacientes en DP fue tratado vía subcutánea, con una frecuencia de una a dos veces por semana en el $75 \%$ de los casos, en tanto que el $84.3 \%$ de los pacientes en hemodiálisis recibió la hormona vía intravenosa 3 veces por semana (una dosis en cada sesión de diálisis). El 87\% del total de niños tratados en DP y HD recibió EPO alfa (Epogen $\left.{ }^{\circledR}\right)$. Las dosis usadas variaron entre 200 y $400 \mathrm{U} / \mathrm{kg} / \mathrm{semana}$, con una clara tendencia a usar mayores dosis a menor edad del paciente, se observó, además, que durante los primeros 24 meses de tratamiento la dosis fue mayor en HD en comparación con DP.

Existe evidencia de que la dosis de EPO puede ser menor en DP que en HD, debido fundamentalmente a que en DP se mantiene una mejor función renal residual, y por lo tanto una mayor capacidad de producir la hormona por las nefronas residuales ${ }^{18,27}$. Igualmente hay estudios respecto a que la dosis requerida es menor al usar la vía subcutánea, lo que corresponde a la práctica clínica habitual en diálisis peritoneal $^{26,33,36}$. 


\section{Complicaciones}

Las complicaciones del tratamiento con EPO han sido descritas en detalle en numerosos estudios. La Hipertensión Arterial (HTA) es una de las más comunes, en niños y adultos, por lo cual existen autores que han recomendado mantener niveles target de hemoglobina/ hematocrito inferiores a las recomendaciones DOQI, sugiriendo una cifra de $10-11 \mathrm{gr} / \mathrm{dl}$ y 30 $35 \%$ para cada uno respectivamente ${ }^{26}$. Se cree que este efecto depende de un aumento en el volumen sanguíneo, en la viscosidad de la sangre, cambios secundarios en la síntesis de óxido nítrico, aumento del calcio intracelular, de la producción de catecolaminas, y a un efecto vasopresor directo dependiente de la hormona. Se han reportado cifras cercanas al 30\% de HTA en tratamientos con esta hormona en pediatría ${ }^{1,19,31}$, y su uso en neonatos debe ser especialmente cauteloso. No parecen existir diferencias entre pacientes en hemodiálisis o peritoneodiálisis.

Resulta especialmente interesante constatar que el aumento del hematocrito después de transfusiones sanguíneas no se asocia a HTA, como tampoco la elevación de la hemoglobina en respuesta al hierro endovenoso ${ }^{37,38}$, existiendo comunicaciones de HTA en relación a la EPO en pacientes con depósitos insuficientes de fierro y previo al aumento de la hemoglobina $^{19,39}$. En los casos en que se observe HTA frente a la introducción de la EPO, su dosificación debe ser reevaluada por el especialista, adecuando la dosis y programando individualmente su ajuste posterior. Se debe considerar el uso de medicamentos antihipertensivos en la fase de HTA, recomendándose el uso de antagonistas del calcio. Finalmente, existen estudios que sugieren que su aplicación subcutánea puede ser menos deletérea que la endovenosa ${ }^{37,38}$.

Otros efectos adversos relacionados con el uso de EPO recombinante humana son: trombosis del circuito extracorpóreo, trombosis de la fístula arteriovenosa, déficit de hierro, hiperkalemia, trombocitosis, hiperfosfemia, cefalea, dolor en el sitio de inyección y síntomas tipo influenza ${ }^{26}$.

\section{Falta de respuesta al tratamiento}

El déficit de hierro es la razón clínica más importante por la cual un paciente puede mostrar ausencia de respuesta a la EPO. Después del inicio de la EPO, es común observar una disminución de los niveles de ferritina sérica y de saturación de transferrina, por lo cual el déficit absoluto o funcional de este ión debe ser cuidadosamente controlado antes y durante el tratamiento con esta hormona. En la próxima sección se analizan en detalle los principales aspectos de esta terapia. Otras causas bien conocidas de falta de respuesta al tratamiento con EPO recombinante, incluyen: infecciones, intoxicación por aluminio, hiperparatiroidismo secundario severo, displasia de la médula ósea, hemólisis y/o excesivas pérdidas sanguíneas que determinan una pseudo-resistencia al uso de EPO, uso de inhibidores de la enzima convertidora de angiotensina que disminuyen la síntesis endógena de EPO y administración de dosis inadecuadas o falta de adherencia al tratamiento ${ }^{1,26}$.

\section{Hierro}

El segundo pilar del tratamiento se basa en reponer los depósitos de hierro y mantenerlos. Este es un punto clave, ya que se sabe que pacientes con déficit de hierro requieren dosis más elevadas de eritropoietina para mantener niveles adecuados de hemoglobina y que la principal causa de respuesta inadecuada a la EPO es el déficit de hierro, especialmente en pacientes en $\mathrm{HD}^{39}$. A pesar de que la administración de preparados orales de hierro es segura y de bajo costo, es inadecuada para mantener depósitos normales de hierro en la mayoría de los pacientes en HD o $\mathrm{DP}^{40}$. Así, por ejemplo, en un estudio prospectivo que incorporó a 25 pacientes adultos en DP crónica se observó que aquellos pacientes que recibieron hierro i.v. Presentaron niveles de hematocrito y de saturación de transferrina significativamente mayores que aquellos pacientes que recibieron tratamiento oral. Adicionalmente la dosis de eritropoietina al final del período de estudio fue significativamente menor en el grupo que recibió tratamiento intravenoso ${ }^{41,42}$. En otro estudio prospectivo cruzado (cross-over) en el que se evaluó la respuesta a la administración oral e intravenosa de hierro dextran en 11 pacientes adultos en CAPD, se detectó una disminución 
rápida de saturación de transferrina y un aumento de las dosis de eritropoietina durante la fase oral del tratamiento, patrón que se mantuvo durante el período de ventana (wash-out). Posteriormente, en la fase parenteral de la terapia, la saturación de transferrina aumentó significativamente permitiendo reducir la dosis de eritropoyetina administrada ${ }^{43}$.

Entre los factores que contribuyen a la mala respuesta a tratamiento oral se encuentran: intolerancia gastrointestinal (constipación, náuseas), una menor absorción intestinal de hierro $^{44-48}$ y baja adherencia al tratamiento, la que es favorecida por el hecho de que los suplementos orales deben ser administrados alejados de medicamentos que estos pacientes reciben de forma rutinaria, tales como quelantes de fósforo y antiácidos.

En resumen, el uso rutinario de una gran cantidad de medicamentos vía oral, sumado a la falta de efectividad de las presentaciones orales de hierro y la actual disponibilidad de preparados intravenosos que se han asociado con una menor frecuencia de eventos adversos, ha determinado, en los últimos años, la realización de un mayor número de estudios respecto al uso de hierro intravenoso en población pediátrica $^{49-51}$.

\section{Vía de administración}

La última recomendación KDOQI postula que algunos pacientes en tratamiento médico y en DP podrían beneficiarse del uso de hierro oral, motivo por el cual no proponen una ruta de administración preferida; por el contrario, en los pacientes en hemodiálisis sugieren que la vía de administración debiera ser intravenosa.

\section{Formas farmacéuticas del Hierro}

\section{Hierro oral}

Las dosis de hierro elemental recomendadas varían entre $2-3 \mathrm{mg} / \mathrm{kg} / \mathrm{día}^{40}$ hasta $6 \mathrm{mg} / \mathrm{kg} /$ día, con un máximo de 150-300 mg diarios, dividido en 2-3 dosis. Debe ser administrado 2 horas antes o 1 hora después de quelantes de fósforo que contienen calcio o de comida, con el fin de aumentar la absorción gastrointestinal. No debe ser adicionado a fórmulas lácteas, ni ingerido en conjunto con cereales, legumbres ni taninos (té, chocolate).

\section{Hierro intravenoso}

La escasez de estudios en población pediátrica se debe a que los preparados iniciales de hierro parenteral, especialmente aquellos asociados con dextran de alto peso molecular, eran mal tolerados y con frecuencia se asociaban con la aparición de eventos adversos, especialmente reacciones de hipersensibilidad que pueden desencadenar anafilaxiaa ${ }^{52-54}$. Se ha descrito que la frecuencia de efectos adversos se asocia con el tipo de hierro parenteral utilizado y con la velocidad de infusión. Motivos adicionales que explicarían la falta de estudios en edades pediátricas incluyen el riesgo de sobrecarga de hierro y la necesidad de contar con una vía venosa segura para su administración, especialmente en pacientes en DP. Aunque muchos clínicos e investigadores han expresado preocupación en relación a la toxicidad de las preparaciones parenterales basados en estudios experimentales de stress oxidativo inducido por hierro ${ }^{55,56}$ y en la asociación descrita en estudios epidemiológicos entre el aumento de la ferritina sérica y mortalidad; no existe hasta este momento evidencia suficiente para eliminar el uso de hierro intravenoso en base a consideraciones de seguridad. En relación al la preocupación acerca de la sobrecarga de hierro, en un estudio realizado en población pediátrica, que comparó 2 formas de administración (intermitente vs mantención) de hierro dextran en pacientes en HD se calculó el riesgo de presentar sobrecarga de hierro (definida como ferritina $>800 \mathrm{ng} / \mathrm{ml}$ o Saturación de transferrina $>50 \%$ ) después de 6 meses de iniciado el tratamiento. Así se determinó que el riesgo era de $20 \%$ en el grupo de pacientes que recibió hierro parenteral de mantención en dosis determinada según niveles de ferritina sérica vs un riesgo de $100 \%$ en el grupo al que se administró dosis intermitentes de hierro intravenoso (10 cursos de hierro dextran en dosis calculada según peso corporal). Es destacable que en este estudio no se aportaron evidencias directas o indirectas de daño orgánico relacionado con sobrecarga de hierro ${ }^{57}$.

Las formulaciones actualmente disponibles de 
hierro parenteral incluyen hierro dextran de bajo peso molecular (infed $\AA$, Cosmofer ${ }^{\circledR}$ ), hierro dextran de alto peso molecular (Dexferrum ${ }^{\circledR}$ ), gluconato férrico sódico (Ferrlecit $\left.{ }^{\circledR}\right)$ y hierro sacarato (Venofer ${ }^{\circledR}$ ). Todas las formas disponibles se han asociado con eventos adversos de tipo inmunológico o bien por liberación a la circulación de hierro bioactivo parcialmente unido, lo que determina stress oxidativo e hipotensión (reacciones de hierro libre o lábil). Las reacciones anafilácticas se observan con mayor frecuencia al utilizar preparaciones con hierro dextran, mientras que las preparaciones sin dextran (gluconato, sacarato) se asocian con reacciones mediadas por liberación de hierro libre a la circulación ${ }^{51,52}$. Las características principales de los diferentes tipos de hierro intravenoso se describen en la tabla 4.

En un estudio en que se comparó la frecuencia y tipo de eventos adversos secundarios a la administración de diferentes fórmulas de hierro parenteral, en base a los registros de la FDA durante el período comprendido entre el año 2001 y 2003; se reportaron un total de 38 reacciones adversas por millón de dosis administradas. En este estudio se observó que la frecuencia de eventos adversos y de reacciones potencialmente fatales era menor en aquellos pacientes que recibieron gluconato férrico sódico o hierro sacarato. Por el contrario, el riesgo total de eventos adversos y de reacciones potencialmente fatales era significativamente mayor en el grupo que recibió preparaciones con hierro dextran de alto peso molecular comparado con los que recibieron preparaciones de bajo peso molecular. Se debe destacar que Venofer® fue la preparación que se asoció con menor número de efectos adversos, y que en el período evaluado, no se registraron casos de paro cardiorrespiratorio ni de reacciones anafilácticas con este medicamento ${ }^{52}$.

\section{Hierro dextran}

Seguridad: En 2 estudios pediátricos en los que se utilizó hierro dextran en 28 pacientes en total, se evidenció un episodio de reacción alérgica que determinó detener la medicación ${ }^{51}$ y 3 eventos adversos auto-limitados que no requirieron suspender el aporte de hierro parenteral (prurito, mialgia y diarrea $)^{42}$. En la actualidad, su uso no está recomendado dado que existen nuevas formulaciones que presentan un mejor perfil de seguridad.

Eficacia: En los 2 estudios mencionados previamente, los que incluyeron pacientes en HD crónica se observó que el uso de hierro dextran es superior al hierro oral para incrementar los depósitos de hierro en pacientes sin déficit inicial de hierro ${ }^{61}$ y que la administración de $4 \mathrm{mg} / \mathrm{kg} / \mathrm{sesión}$ durante 10 sesiones consecutivas de HD (dosis máxima de $100 \mathrm{mg}$ ) en pacientes con déficit de depósitos de hierro permitió aumentar significativamente los nive-

Tabla 4. Características de los diferentes tipos de hierro para uso intravenoso

\begin{tabular}{llll}
\hline & \multicolumn{1}{c}{$\begin{array}{c}\text { Hierro dextran } \\
\text { (Infed) }\end{array}$} & \multicolumn{1}{c}{$\begin{array}{c}\text { Sacarato de hierro } \\
\text { (Venofer) }\end{array}$} & \multicolumn{1}{c}{$\begin{array}{c}\text { Gluconato férrico } \\
\text { (Ferrilecit) }\end{array}$} \\
\hline Peso molecular kD & $73-103$ & $34-60$ & $289-440$ \\
Vida media & $40-60 \mathrm{hr}$ & $6 \mathrm{horas}$ & $1 \mathrm{hora}$ \\
Volumen distribución & $6 \mathrm{tt}$ & $3,2-7,3 \mathrm{lt}$ & $6 \mathrm{lt}$ \\
Eliminación renal & $\mathrm{no}$ & $<5 \%$ & insignificante \\
Presentación & $100 \mathrm{mg} / 2 \mathrm{ml}$ & $100 \mathrm{mg} / 5 \mathrm{ml}$ & $62,5 \mathrm{mg} / 5 \mathrm{ml}$ \\
Test primera dosis & $\mathrm{si}$ & $\mathrm{no}$ & $\mathrm{no}$ \\
EV directo & $100 \mathrm{mg} \mathrm{en} 2 \mathrm{~min}$ & $100 \mathrm{mg} \mathrm{en} 5 \mathrm{~min}$ & $125 \mathrm{mg} 10 \mathrm{~min}$ \\
Infusión EV & $\mathrm{no}$ & $100 \mathrm{mg} / 100 \mathrm{cc} \mathrm{SF}$ pasar & $125 \mathrm{mg} / 100 \mathrm{cc} \mathrm{SF}$ \\
& & $\mathrm{al} \mathrm{menos} \mathrm{en} \mathrm{15min}$ & pasar en $1 \mathrm{~h}(2 \mathrm{mg} / \mathrm{min})$ \\
Dosis habitual máxima por vez & $100-1000 \mathrm{mg}$ & $100 \mathrm{mg}$ & $125 \mathrm{mg}$ \\
Reacción anafiláctica grave & $\mathrm{si}$ & $\mathrm{no}$ & $\mathrm{no}$ \\
\hline
\end{tabular}


les de hematocrito y disminuir significativamente la dosis de EPO utilizada, dos meses después de completado el tratamiento ${ }^{49}$.

\section{Gluconato Férrico Sódico: Ferrlecit ${ }^{\circledR}$}

Seguridad: En un estudio randomizado, doble ciego y prospectivo, que incluyó 66 pacientes pediátricos en HD crónica, en el cual se comparó la eficacia de dos esquemas de administración de este medicamento $(1,5 \mathrm{mg} / \mathrm{kg}$ vs 3 $\mathrm{mg} / \mathrm{kg}$ ) durante 8 sesiones consecutivas de HD, se observaron efectos adversos en 2 pacientes. Uno de los pacientes estaba recibiendo hierro i.v. En bajas dosis y presentó síntomas gastrointestinales y el segundo paciente presentó anemia severa cuando se encontraba en tratamiento con $3 \mathrm{mg} / \mathrm{kg} / \mathrm{sesión}$. No se detectaron reacciones anafilácticas, alérgicas ni muertes durante el período evaluado ${ }^{58}$. En un estudio pequeño que incluyó 8 pacientes en HD crónica, se administró Gluconato Férrico $1 \mathrm{mg} / \mathrm{kg} / \mathrm{se}$ manal por 12 semanas sin observarse efectos adversos $^{51}$. Finalmente, en un estudio en el que se administraron 60 dosis de gluconato férrico a un total de 13 pacientes en HD o trasplantados renales, se observó una reacción adversa autolimitada consistente en dolor abdominal severo, vómitos y parestesias de los dedos. En este último estudio la dosis promedio fue de $3 \pm 2$ $\mathrm{mg} / \mathrm{kg}$, con un rango de 1,5-8,8 $\mathrm{mg} / \mathrm{kg}$ y la reacción descrita la presentó el paciente que recibió la dosis más elevada ${ }^{59}$.

Eficacia: A la fecha, el estudio prospectivo, randomizado, que incluyó a un mayor número de pacientes pediátricos es el publicado por Warady y cols., en el que se evaluó a un grupo de pacientes en HD. Al ingreso al estudio, todos los pacientes cumplían uno o ambos criterios de deficiencia de hierro en pacientes en tratamiento con agentes estimuladores de eritropoiesis (TSAT $<20 \%$ y/o ferritina $<100 \mathrm{ng} /$ $\mathrm{ml})$. En todos los pacientes evaluados se detectó un aumento significativo de Hb, saturación de transferrina, ferritina sérica y concentración de hemoglobina en reticulocitos. Adicionalmente, la dosis de eritropoyetina fue disminuida en 5 de 56 pacientes.

En este mismo estudio se observó que dosis de $1,5 \mathrm{mg} / \mathrm{kg}$ y de $3 \mathrm{mg} / \mathrm{kg}$ por sesión de HD durante 8 sesiones consecutivas, eran igual- mente efectivas en términos de aumentar los índices hematológicos asociados con deficiencia de hierro ${ }^{58}$.

\section{Hierro Sacarato: Venofer ${ }^{\circledR}$}

Seguridad: Existen escasos datos de seguridad en población pediátrica y ninguno de ellos fue diseñado para evaluar la frecuencia de reacciones adversas. En 2 estudios pediátricos que incluyeron a 33 pacientes en hemodiálisis no se reportaron reacciones adversas ${ }^{49,60}$.

Eficacia: En uno de los estudios mencionados se analizó de forma prospectiva la respuesta a diferentes dosis de hierro sacarato en 14 pacientes pediátricos en hemodiálisis. Los pacientes se dividieron en 3 grupos; el grupo A estaba formado por los que presentaban déficit de fierro y recibieron hierro intravenoso en dosis de $3 \mathrm{mg} / \mathrm{kg} / \mathrm{sesión}$, el grupo B estaba formado por pacientes sin déficit de fierro y recibieron $0,3 \mathrm{mg} / \mathrm{kg}$ de hierro sacarato por sesión y el grupo $\mathrm{C}$ formado por aquellos con posible sobrecarga de fierro (ferritina $>400 \mathrm{ng}$ / $\mathrm{ml})$ no recibieron tratamiento con hierro. Se debe destacar que en los pacientes del grupo A fue necesario reducir la dosis a $1 \mathrm{mg} / \mathrm{kg} / \mathrm{sesión}$ dado que presentaron un marcado aumento de los niveles de ferritina. Los autores concluyeron que dosis de $1 \mathrm{mg} / \mathrm{kg} / \mathrm{sesión} \mathrm{y} \mathrm{de} 0,3 \mathrm{mg} / \mathrm{kg} /$ sesión eran adecuados para terapia de corrección y mantención, respectivamente ${ }^{60}$.

El primer estudio randomizado controlado que evaluó el uso de hierro intravenoso en pacientes con ercr en diálisis peritoneal incluyó a 126 pacientes adultos en tratamiento con eritropoyetina. Los pacientes que recibieron hierro intravenoso presentaron un incremento significativamente mayor en los niveles de hemoglobina que aquellos que no recibieron hierro adicional (IC 95\% 0,3-1,2; p = 0,0028), lo mismo sucedió con los niveles de ferritina sérica y saturación de transferrina. En este estudio se demostró que el uso de Venofer ${ }^{\circledR}$ en asociación con eritropoietina, en pacientes en DP aumentaba los niveles de hemoglobina, reducía la necesidad de realizar intervenciones para el tratamiento de la anemia (transfusiones, aumentar dosis de eritropoietina o administrar dosis adicionales de hierro intravenoso) y permitía reponer los depósitos de hierro ${ }^{61}$. 


\section{Dosificación}

No existe actualmente un consenso respecto a la dosis y frecuencia de administración de hierro intravenoso en población pediátrica ${ }^{3,62}$. La dosificación apropiada es un tema complejo de establecer, principalmente porque existen escasos estudios realizados en población pediátrica, la mayoría de los cuales incorporan un número pequeño de pacientes y fueron realizados en pacientes en hemodiálisis. Por otra parte, la dosis a administrar depende de la forma farmacéutica que se esté utilizando. Por lo que si al hecho de que existen pocos estudios en población pediátrica, agregamos que éstos han sido realizados con distintas formas de hierro intravenoso y que en su mayoría han evaluado pacientes en hemodiálisis en distintos estados de depósitos de hierro, no es posible entregar pautas generales de dosificación.

Adicionalmente, existen escasos estudios en pediatría dirigidos específicamente al tema del tratamiento parenteral de mantención. En un estudio de tipo transversal se incluyó a 19 pacientes en hemodiálisis crónica, a quienes se les administró hierro sacarato (Venofer ${ }^{\circledR}$ ) en distintas dosis dependiendo de si existía o no déficit de fierro. En aquellos pacientes sin déficit de fierro se administró una dosis de mantención de $2 \mathrm{mg} / \mathrm{kg} / \mathrm{semanal}$ (máximo $100 \mathrm{mg} / \mathrm{semana}$ ) y a los pacientes que presentaban déficit de fierro se indicó una dosis de $7 \mathrm{mg} / \mathrm{kg}$ por una vez (máximo 200 mg) y luego una dosis de mantención de $2 \mathrm{mg} / \mathrm{kg} / \mathrm{semanal}$ (máximo $100 \mathrm{mg}$ ). Debido al escaso número de pacientes incluidos, al carácter transversal del estudio, a que no aporta datos respecto a variaciones en los índices de evaluación de depósitos de fierro con terapia intermitente y de mantención; no es posible, en base a este estudio, establecer el régimen más apropiado de administración de hierro intravenoso en pediatría ${ }^{50}$.

A pesar de lo mencionado previamente, se debe destacar que en estudios realizados en pacientes en HD, se recomienda que en situaciones de déficit de hierro los pacientes reciban una dosis de carga que varía entre $5-7 \mathrm{mg} / \mathrm{kg}$ con un máximo de 100-200 mg seguida por una dosis de mantención que varía entre $1-5 \mathrm{mg} / \mathrm{kg} /$ semana hasta alcanzar los niveles deseados de $\mathrm{Hb}$, ferritina y saturación de transferrina ${ }^{50,51,58}$.
Otros autores recomiendan la administración de hierro intravenoso durante 8 a 10 sesiones consecutivas de HD en dosis que varían entre 1-4 mg/Kg/sesión, en aquellos pacientes con déficit de hierro ${ }^{49,59-61}$.

En nuestra experiencia de los últimos 5 años, en una población de 51 pacientes en DP, la aplicación de hierro sacarato (Venofer®) en dosis de $7 \mathrm{mg} / \mathrm{kg} /$ dosis con un máximo de 100 mg por semana por 3 semanas consecutivas, en pacientes con niveles de ferritina menor a 100 ng/ml, ha mostrado ser suficiente para recuperar los depósitos de fierro a un target de 100$500 \mathrm{ng} / \mathrm{ml}$ de ferritina sérica, un mes después de iniciado el tratamiento. En el período indicado sólo hemos observado vómitos aislados en un paciente de 2 años de edad, los que cedieron rápidamente al suspender la terapia (observaciones no publicadas).

\section{Administración de hierro intravenoso e infección}

Bajo condiciones normales, el hierro libre está prácticamente ausente en los tejidos, con concentraciones de hasta $10^{-18}$ y las proteínas titulares que unen hierro impiden que el hierro libre circule en la sangre donde puede actuar como factor de crecimiento bacteriano y alterar los mecanismos de defensa del organismo (reduce quimiotaxis de polimorfonucleares, disminuye la fagocitosis y la destrucción intracelular de microorganismos). Aunque la literatura existente no es concluyente respecto a la asociación entre la administración de hierro e infección; parece razonable evitar la sobrecarga de hierro y no administrar hierro intravenoso en el contexto de infecciones activas ${ }^{63,64}$.

\section{Conclusiones}

La anemia es un problema de gran relevancia en los pacientes pediátricos portadores de enfermedad renal crónica. Su manejo ha mejorado notablemente en la última década, sin embargo, sigue siendo un problema de alta prevalencia en este grupo de enfermos. La perspectiva del pediatra difiere significativamente de la del médico internista, por lo que se hace imprescindible contar con pautas de diagnóstico, 
evaluación y tratamiento dirigidas específicamente a la población infantil. En este sentido, la mayor parte de las recomendaciones actuales provienen de estudios realizados en adultos, las cuales se han ajustado para ser utilizadas en pacientes pediátricos. En la actualidad, la terapia se basa fundamentalmente en la utilización de agentes estimuladores de la eritropoiesis y en el aporte de suplementos de hierro, los cuales deben ser utilizados en un contexto de estrecha vigilancia médica para alcanzar el efecto deseado evitando las potenciales reacciones adversas de estas terapias. En el futuro será posible contar con medicamentos de efecto más prolongado y menores reacciones secundarias, lo cual contribuirá a mejorar el nivel de vida de estos pacientes durante la etapa pretrasplante de la enfermedad renal crónica. La situación nacional no difiere de la reportada en otros países, así, el tratamiento del paciente en hemodiálisis está considerado en las guías GES (Garantías Explícitas en Salud) del Ministerio de Salud, sin embargo, su diseño, al igual que el de las guías KDOQI, está basado en estudios realizados en adultos, por lo que se requieren estudios multicéntricos prospectivos en población pediátrica que permitan proponer recomendaciones generales para el uso de hierro intravenoso en la población mencionada.

\section{Referencias}

1.- Fine $R$, Whyte $D$, Boydstun I: Conservative management of chronic renal insufficiency. In Avner E, Harmon W, Niaudet P. Pediatric Nephrology. Fifth edition. Lippincott Williams and Wilkins; Philadelphia, USA, 2004: 1298-300.

2.- Koshy S, Geary D: Anemia in children with chronic kidney disease. Pediatr Nephrol 2008; 23: 209-19.

3.- K/DOQI clinical practice guidelines and clinical practice recommendations for anemia in chronic kidney disease. Am J Kidney Dis 2006; 47 (S3): S11-S145.

4.- Gerson A, Hwang W, Fiorenza J, Barth K, Weiss L, Zelikovsky $N$ : Anemia and health-related quality of life in adolescents with chronic kidney disease. Am J Kidney Dis 2004; 44: 1017-23.

5.- Frank H, Heusser K, Höffken B, Huber P, Schmieder $R E$, Schobel HP: Effect of erythropoietin on cardiovascular prognosis parameters in hemodialysis patients. Kidney Int 2004; 66 (2): 832-40.

6.- Temple RM, Deary IJ, Winney RJ: Recombinant erythropoietin improves cognitive function in patients maintained on chronic ambulatory peritoneal dialysis. Nephrol Dial Transplant 1995; 10 (9): 1733-8.

7.- Morris KP, Sharp J, Watson S, et al: Non-cardiac benefits of human recombinant erythropoietin in endstage renal failure and uremia. Arc Dis Child 1993; 69: $580-6$

8.- Jacobs C, Frei D, Perkins A: Results of the European Survey on Anemia Management 2003 (ESAM 2003): current status of anemia management in dialysis patients, factors affecting epoetin dosage and changes in anaemia management over the last 5 years. Nephrol Dial Transplant 2005; 20 (Suppl 3): iii3-iii24.

9.- Rama de Nefrología de la Sociedad Chilena de Pediatría: Enfermedad renal crónica Registro Nacional 2006. Congreso conjunto de las sociedades de Nefrología, Hipertensión y Transplante; Septiembre 2007, Pucón, Chile.

10.- Warady B, Holloway M: Presentado en 27 th Annual Dialysis Conference Denver, Colorado, USA, University of Missouri, Febrero 2007.

11.- North American Pediatric Renal Transplant Cooperative Study (2006) Annual report: Disponible en http:/ /web.emmes.com/study/ped/annlrept/annlrept2006.pdf

12.- Ma JZ, Ebben J, Xia H, Collins AJ: Hematocrit level and associated mortality in hemodialysis patients. J Am Soc Nephrol 1999; 10 (3): 610-9.

13.- Xia H, Ebben J, Ma JZ, Collins AJ: Hematocrit levels and hospitalization risks in hemodialysis patients. J Am Soc Nephrol 1999; 10 (6): 1309-16.

14.- Warady B, Ho M: Morbidity and mortality in children with anemia at initiation of dialysis. Pediatr Nephrol 2003; 18: 1055-62.

15.- Mitsnefes M, Kimball TR, Border WL, Witt SA, Glascock $B J$, Khoury PR, Daniels SR: Impaired left ventricular diastolic function in children with chronic renal failure. Kidney Int 2004; 65: 1461-6.

16.- Mitsnefes M, Daniels S, Schwartz S, Meyer R, Khoury $P$, Strife $F$ : Severe left ventricular hypertrophy in pediatric dialysis: prevalence and predictors. Pediatr Nephrol 2000; 14: 898-902.

17.- K/DOQI; National Kidney Foundation (2006) III Clinical Practice Recommendations for anemia in Chronic Kidney Disease in Children. Am J Kidney Dis 47 (Suppl 3): S (86-108).

18.- Neu AM, Ho PL, mcdonald R: Chronic Dialysis in Children and Adolescents. The 2001 Annual Report of the North American Pediatric Renal Transplant Cooperative Study. Pediatr Nephrol 2002; 17: 65663.

19.- Yorgin P, Al-Uzri A: Management of Renal Anemia, en: Warady B, Fine R, Schaeffer F, Alexander S: Pediatric Dialysis, Kluber Academic Publishers, Dordretch,Boston, London, 2004: pp 295-331.

20.- Fishbane Maesaka J: Iron management in End Stage Renal Disease. Am J K Dis 1997; 29: 319-33.

21.- Nissenson A: Achievieng target hematocrit in dialysis patients: new concept in iron management. Am J K Dis 1997; 30: 907-11.

22.- Lai PH, Everett R, Wang F, Arakawa T, Golwasser E: Structure Characterization of human Erythropoietin. 
J Biol Chem 1986; 261: 3116-21.

23.- Erslev A: Humoral regulation of red cell production. Blood, 1953; 8: 349-57.

24.- Caro J, Erslev AJ: Erythropoietin assays and their use in the study of anemias. Contrib Nephrol 1988; 66: 54.

25.- Schwartz E: The Anemias, en: Behrman R, Kliegman $\mathrm{R}$, Jenson H, eds. Nelson Textbook of Pediatrics, 16th Ed., Philadelphia;WB Saunders Company 2000: 146172.

26.- Van Damme-Lombaerts R, Herman J: Erithropoietin treatment in children with renal failure. Pediatr Nephrol 1999; 13: 148-52.

27.- Singh A, Szczech L, Tang K, Barnhart H, Sapp S, Wolfson $M$, Reddan D: Correction of anemia with Epoetin Alfa in Chronic Kidney Disease. N Engl J Med 2006; 355: 2085-98.

28.- Macdougal IC: An overview of the efficacy and safety of novel erythropoiesis stimulating protein (NESP). Nephrol Dial Transplant 2001; 16 (s3): 14-21.

29.- Kessler M, Hannedouche T, Fitte H, Cayotte J, Urena $P$, Reglier J: Darbepoietin-alfa treatment of anemia secondary to chronic renal failure in dialysis patients: results of a French multicenter study. Nephrol Ther 2006; 2 (4): 191-9.

30.- Bock A, Hirt-Minkowski P, Brunisholz M, Keusch G, Rey S, Von Albertini B: Darbepoetin Alpha in lowerthan-equimolar doses maintains hemoglobin levels in stable hemodialysis patients converting from epoetin alpha/beta. Nephrol Dial Transplant 2008; 23 (1): 301-8.

31.- Locatelli F, Reigner B: C.E.R.A.: pharmacodynamics, pharmacokinetics and efficacy in patients with chronic kidney diseases. Expert Opin Invest Drugs 2007; 16 (10): 1649-61.

32.- Sulowicz W, Locatelli F, Ryckelynck JP, Balla J, Csiky $B$, Harris K, Ehrhard P, Beyer U: Once-monthly subcutaneous C.E.R.A. maintains stable hemoglobin control in patients with chronic kidney diseases on dialysis and converted directly from epoetin one to three times weekly. Clin J Am Soc Nephrol 2007; 2 (4): 637-46.

33.- K/DOQI clinical practice guidelines and clinical practice recommendations for anemia in chronic kidney disease. Am J Kidney Dis 2006; 47 (S3): S11-S145.

34.- Locatelli F, Aljama P, Barany P: Revised European best practice guidelines for the management of anaemia in patients with chronic renal failure. Nephrol Dial Transplant 2004; 19 (S2): S1-S47.

35.- Brant JL: Safety and efficacy of Erythropoietin in children with chronic renal failure. Pediatr Nephrol 1999; 13: 143-7.

36.- K/DOQI clinical practice guidelines for anemia of chronic kidney disease: update 2000. Am J Kidney Dis 2001; 37 (1): S182-S238.

37.- Buckner FS, Eschbach J, Haley N., Davison R, Adamson J: Hypertension following erythropoietin therapy in anemic hemodialysis patients, Am J Hypertens 1990; 3 (12): 947-55.

38.- Vaziri ND: Cardiovascular effects of erythropoietin and anemia correction. Curr Opin Nephrol and Hypertens 2001; 10 (5): 633-7.

39.- Li S, Foley RN, Gilbertson DT, Liu J, Collins AJ: Clinical factors associated with achieving K/DOQI hemoglobin targets in hemodialysis patients. Int Urol Nephrol 2003; 35 (3): 399-405.

40.- Schröder C: The management of anemia in pediatric peritoneal dialysis patients. Pediatr Nephrol 2003; 18: 805-9.

41.- Ahsan $N$ : Intravenous infusion of total dose iron is superior to oral iron in treatment of anemia in peritoneal dialysis patients: a single center comparative study. J Am Soc Nephrol 1998; 9: 664-8.

42.- Warady B, Kausz A, Lerner G, Brewer E, Chadha V, Brugnara C, Dahl N, Watkins S: Iron therapy in the pediatric hemodialysis population. Pediatr nephrol 2004; 19: 655-61.

43.- Ahsan $N$ : Infusion of total dose iron versus oral iron supplementation in ambulatory peritoneal dialysis patients: a prospective, cross-over trial. Adv Perit Dial 2000; 16: 80-4.

44.- Kooistra MP, Niemantsverdriet EC, van Es A, MolBeermann NM, Struyvenberg A, Marx JJ: Iron absorption in erythropoietin-treated haemodialysis patients: effects of iron availability, inflammation and aluminium. Nephrol Dial Transplant 1998; 13 (1): 82-8.

45.- Fishbane S, Maesaka JK: Iron management in endstage renal disease. AJKD 1997; 29(3): 319-33.

46.- Johnson DW: Intravenous versus oral iron supplementation in peritoneal dialysis patients. Perit Dial Int 2007; 27 (Suppl 2): S225-60.

47.- Valderrábano F: Erythropoietin in chronic renal failure. Kidney Int 1996; 50: 1373-91.

48.- Fishbane S. Iron treatment: Impact of safety issues. AJKD 1998; 32(6, Suppl 4): S152-S156.

49.- Greenbaum L, Pan C, Caley C, Nelson T, Sheth K: Intravenous iron dextran and erythropoietin use in pediatric hemodialysis patients. Pediat Nephrol 2000; 14: 908-11.

50.- Morgan H, Gautam M, Geary D: Maintenance intravenous iron therapy in pediatric hemodialysis patients. Pediatr Nephrol 2001; 16: 779-83.

51.- Tenbrock K, Müller-Berghaus J, Michalk D, Querfeld $U$ : Intravenous iron treatment of renal anemia in children on hemodialysis. Pediatr Nephrol 1999; 13: 580-2.

52.- Chertow G, Mason P, Vaage-Nilsen O, Ahlmé: Update on adverse drug events associated with parenteral iron. Nephrol Dial Trnsplant 2006; 21: 378-82.

53.- Faich G, Strobos J: Sodium ferric gluconate complex in sucrose: safer intravenous iron therapy than iron dextran. Am J Kidney Dis 1999; 33 (3): 464-70.

54.- Fishbane S: Safety in iron management. Am J Kidney Dis 2003; 41 (S5): S18-S26.

55.- Zager R, Johnson A, Hanson S: Parenteral iron nephrotoxicity: Potencial mechanisms and consequences. Kidney Int 2004; 66: 144-56.

56.- Agarwal R, Vasavada $N$, Sachs $N$, Chase S: Oxidative stress and renal injury with intravenous iron in patients 
with chronic kidney disease. Kideny Int 2004; 65: 2279-89.

57.- Ruiz-Jaramillo M, Guízar-mendoza J, Gutiérrez-Navarro $M$, Dubey-Ortega $L$, Amado-Licoma $N$ : Intermittent versus maintenance iron therapy in children on hemodialysis: a randomized study. Pediatr nephrol 2004; 19: 77-81.

58.- Warady B, Zobrist R, Wu J, Finan E: Sodium ferric gluconate complex therapy in anemic children on hemodialysis. Pediatr Nephrol 2005; 20: 1320-7.

59.- Yorgin P, Belson A, Sarwal M, Alexander S: Sodium ferric gluconate therapy in renal transplant and renal failure patients. Pediatr nephrol 2000; 15: 171-5.

60.- Leijin E, Monnenes LA, Cornelissen EA: Intravenous iron supplementation in children on hemodialysis. J
Nephrol 2004; 17 (3): 423-6.

61.- Singh H, Reed J, Noble S, Cangiano J, Van Wick D for the United Status Iron Sucrose (Venofer) Clinical Trial Group: Effect of intravenous iron sucrose in peritoneal dialysis patients who receive erithropoiesisstimulating agents for anemia: a randomized, controlled trial. Clin J Am Soc Nephrol 2006; 1: 475-82.

62.- Gillespie $R$, Wolf $F$ : Intravenous iron therapy in pediatric hemodialysis patients: a meta-analysis. Pediatr Nephrol 2004; 19: 662-6.

63.- Adamson J, Cavill I, Fishbane S, Petersen J, Wish J: Seminars in dialysis 1999; 12 (3): 182-94.

64.- Brewster U, Perazella M: Intravenous iron and the risk of infection in End-Stage Renal Disease patients. Seminars in dialysis 2004; 17 (1): 57-60. 